\title{
The Arm Profile Score: A new summary index to assess upper limb movement pathology
}

\author{
Ellen Jaspers ${ }^{\mathrm{a}, *}$, Hilde Feys ${ }^{\mathrm{a}}$, Herman Bruyninckx ${ }^{\mathrm{b}}$, Katrijn Klingels ${ }^{\mathrm{a}}$, \\ Guy Molenaers ${ }^{\mathrm{c}, \mathrm{d}}$, Kaat Desloovere ${ }^{\mathrm{a}, \mathrm{d}}$ \\ ${ }^{a}$ Department of Rehabilitation Sciences, Faculty of Kinesiology and Rehabilitation Sciences, Katholieke Universiteit Leuven, Belgium \\ ${ }^{\mathrm{b}}$ Department of Mechanical Engineering, Faculty of Engineering, Katholieke Universiteit Leuven, Belgium \\ c Orthopaedics Section, Department of Musculoskeletal Sciences, Katholieke Universiteit Leuven, Belgium \\ d Clinical Motion Analysis Laboratory, CERM, University Hospital Pellenberg, Belgium
}

\section{A R T I C L E I N F O}

Article history:

Received 15 November 2010

Received in revised form 28 April 2011

Accepted 5 May 2011

\section{Keywords:}

Upper limb movement pathology

Summary index

Kinematics

Arm Profile Score

Arm Movement Analysis Profile

\begin{abstract}
A B S T R A C T
Although three-dimensional movement analysis is being increasingly used to evaluate upper limb (UL) movements, information on how to interpret the complex data is still missing. This paper introduces a new summary index, the "Arm Profile Score" (APS), to evaluate the severity of UL movement pathology based on kinematic data, similar to the "Gait Profile Score". The APS is calculated from the root mean square (RMS) difference between kinematic data of the individual child with UL movement deficits and average data from typically developing children. The APS can be decomposed into 13 Arm Variable Scores (AVS), representing the different joint angles. The APS, together with the AVSs form the "Arm Movement Analysis Profile" (A-MAP).

Face and construct validity were established for eight UL tasks in a group of 20 children with hemiplegic cerebral palsy (HCP). Intra-session variability was low for the different tasks, with median inter-quartile ranges below $2^{\circ}$. Correlation analysis showed few significant correlations between the individual AVSs and between the AVS and APS, implying that the A-MAP provides considerably more information compared to the APS only. The APS also showed good correlations with the House classification, and with measures of muscle tone, manual muscle strength and grip strength.

This study provides a sound base to use the APS to evaluate UL movement pathology in children with HCP. Further study will need to confirm its value as an outcome measurement.
\end{abstract}

(c) 2011 Elsevier B.V. All rights reserved.

\section{Introduction}

In children with hemiplegic cerebral palsy (HCP), basic upper limb (UL) functions such as reach, grasp and release objects are often compromised. To improve their functionality and independence in daily life, a well-targeted treatment planning based upon the child's individual needs is crucial. Available clinical assessments rely on the therapist grading the child's severity of UL movement pathology, often based on visual observation and ordinal scales [1]. The use of objective methods might contribute to a better understanding of the pathological movement patterns seen in children with HCP.

Although three-dimensional movement analysis (3DMA) is being increasingly used to evaluate UL movements in children with $\mathrm{CP}[2-4]$, there is a lack of consensus regarding the biomechanical

\footnotetext{
* Corresponding author at: Tervuursevest 101, 3001 Leuven, Belgium. Tel.: +32 16 329117; fax: +32 16329197.

E-mail address: ellen.jaspers@faber.kuleuven.be (E. Jaspers).
}

model, and which tasks to analyze [4]. We therefore proposed a measurement procedure for UL-3DMA, including the recommendations from the International Society of Biomechanics (ISB) [5]. Given the variety of UL functions, a comprehensive set of tasks was selected to represent clinically relevant activities for children with $\mathrm{HCP}$. The procedure was proven reliable in typically developing children (TDC) [6] and children with HCP [7]. To establish its true value as a clinical tool, a next step is to further expand our understanding of the information obtained from this 3DMA.

For gait analysis, kinematic data are typically reported as graphs which are used to guide the clinical decision-making or to evaluate treatment outcomes. Nonetheless, the interpretation of the highly interdependent data remains complex and requires numerous clinical expertise. Various comprehensive measures have therefore been proposed to quantify the overall severity of gait pathology, i.e. the Gillette Gait Index (GGI) [8], the Gait Deviation Index (GDI) [9] and the Gait Profile Score (GPS) [10]. While the GGI incorporates spatial, temporal and kinematic parameters, the GDI and GPS are based solely on kinematic data. The GDI is derived from gait feature analysis, which requires a large dataset of subjects with a wide 
range of gait pathologies. Conversely, the GPS is independent of the feature analysis and can be calculated directly from the data of one individual and the averaged data of typical people.

Given that the construction of the GPS is well applicable for other set-ups, it seems interesting to compose a similar single outcome measure to summarize overall UL pathology. A recent study by Riad et al. has described the "Arm Posture Score" as an index to score the deviating arm posture of children with HCP during gait [11]. However, an index to assess the amount of deviating joint movement during the execution of functional UL tasks is still missing. This paper therefore proposes the "Arm Profile Score" (APS) as a summary index of UL movement pathology in children with HCP. Face and construct validity are presented through the construction of the index and by relating it with other UL measurements.

\section{Method}

\subsection{Participants}

Twenty children with HCP (11 boys, 9 girls; age 10.9 ys \pm 2.9 ys) and 20 individually age-matched TDC with no history of musculoskeletal or neurological problems (11 boys, 9 girls; age 10.9 ys \pm 3.0 ys) were selected. Children with HCP were included if they met following inclusion criteria: (1) diagnosis of congenital HCP; (2) aged 5-15 years; (3) minimum ability to actively grasp an object and hold it weakly (House 4) [12]. Children were excluded in case of UL surgery or BTX-injections 6 months prior to testing. Ethical approval was granted by the University Hospital Ethics Committee. Written informed consent was signed by all children's parents.

Ten children with HCP had a right and 10 a left hemiplegia. They were sampled across the first three levels of the Manual Ability Classification Scale (MACS) [13] (level I: 4 children; level II: 15 children, level III: 1 child).

\subsection{Data collection}

All assessments were performed by one trained physiotherapist, at the clinical motion analysis laboratory (University Hospital Pellenberg, Belgium), using a standardized protocol for the UL-3DMA [6,7] and the clinical evaluation [14].

\subsubsection{UL-3DMA [6,7]}

Data were collected with the Vicon MX-system (Oxford Metrics Group, UK) and filtered using spline-interpolation [15]. Kinematics were calculated following the ISB-recommendations [5].

The movement protocol consisted of three reach tasks (forwards, upwards, sideways); two reach-to-grasp tasks with changing handgrip (forearm pronation or neutral rotation); and three gross motor tasks (hand to mouth, hand to head, hand to contralateral shoulder). During task execution, children were seated in a custommade chair with individually adjustable reaching distance/height and sitting position. Children executed every task with their non-preferred arm at self-selected speed, i.e. the hemiplegic arm in children with HCP and the non-dominant arm in TDC. Within and between session reliability of the protocol has been established in both groups $[6,7]$.

\subsubsection{Clinical evaluation [14]}

Passive range of motion (PROM) was assessed at the shoulder (flexion/abduction/ internal-external rotation), elbow (extension/supination) and wrist (extension with fingers flexed/extended), with a universal goniometer. All joint movements were graded dichotomously ( $>10^{\circ}$ decreased PROM) and a total score and sub-scores were calculated. Muscle tone was evaluated with the Modified Ashworth Scale (MAS; 6point ordinal scale) [16] for the shoulder (adductors/extensors/internal-external rotators), elbow (flexors/extensors/pronators), and wrist and finger (flexors). A total score and sub-scores for each of the muscle groups were calculated. Muscle strength was scored with the Manual Muscle Test (MMT; 8-point ordinal scale) [17]. A total score for muscle strength was calculated, along with sub-scores for the shoulder (flexors/abductors/adductors), elbow (flexors/extensors), forearm (pronators/supinators), and wrist (flexors/extensors). Grip strength was evaluated with a Jamardynamometer. The ratio of the mean of three maximum voluntary contractions of the hemiplegic versus the non-hemiplegic hand was calculated. Interrater and test-retest reliability of these assessments have been established in children with HCP [14]. The House classification [12] was used to evaluate the functional use of the hemiplegic UL. This 9-level scoring system ranges from non-use of the hemiplegic arm and hand, to a completely spontaneous arm and hand use. The reliability of this classification has been established in a previous unpublished study in 30 children with $\mathrm{HCP}$ (interrater ICC 0.86; test-retest ICC 0.96).

\subsection{Data reduction}

Each task was repeated four times within one single trial. Three successful trials were collected for every task, producing 12 movement cycles per task. One movement cycle was defined from 'hand on ipsilateral knee' to the 'point of task achievement' (PTA) [18]. The second and third cycle of every trial was identified based on frame-by-frame inspection of the moving markers, resulting in six cycles per task.

Kinematics for the trunk, scapula, shoulder, elbow and wrist were calculated with BodyMech (www.bodymech.nl) and additional custom-written routines (Matlab) $[6,7,19]$. Movement cycles were time-normalized and visualized as a function of time for every joint angle. Erroneous signals due to artefacts caused by marker-occlusion were removed from the dataset. Next, the root mean square error (RMSE) of each cycle versus the mean of the remaining cycles per task was calculated per child. The three movement cycles with lowest RMSE were considered to represent the most consistent task performance and were retained for the construction of the index (see flowchart Fig. 1).

2.4. Construction of the Arm Variable Score (AVS), the Arm Profile Score (APS) and the Arm Movement Analysis Profile (A-MAP)

The Arm Variable Score (AVS), Arm Profile Score (APS) and Arm Movement Analysis Profile (A-MAP) were calculated following the mathematical construction of the GPS, as described by Baker et al. [10].

The AVS was calculated for every joint angle as the RMSE between the point-bypoint comparison of the joint angle of the hemiplegic child and that same joint angle of the reference database. The reference database was derived from the kinematic data of the 20 individually age-matched TDC. The AVS gives an index of deviation for a single joint angle. The RMSE-average of all 13 joint angles equals the APS, an index that summarizes the overall severity of UL movement pathology for each of the eight tasks. Next, the APS and 13 AVS-scores were averaged over the three movement cycles per task and presented together in a bar chart, creating the "Arm Movement Analysis Profile" (A-MAP, Fig. 2). The A-MAP thus provides insight into the contribution of each variable to an elevated APS. Finally, a Global-APS (one index) and concurrent Global-AVSs were calculated as an average index for all eight tasks.

\subsection{Validation of the AVS and APS}

The APS is a Euclidian distance measure, likely to have a Chi-square distribution. Results are therefore reported in terms of median values and inter-quartile ranges (IQR) [10].

Intra-session variability was calculated as the median IQR of the APS for each child, estimated from the three movement cycles. To investigate the properties of the individual AVS-scores, a Spearman-rank correlation was calculated between each AVS and the APS, and for each pair of AVSs.

Spearman-rank correlation coefficients were calculated between the APS/ AVSs and the House-scores for each task and between the APS and the tota scores and sub-scores of PROM, muscle tone, manual muscle strength and grip strength.

Correlation coefficients $>0.70$ were considered to indicate high, $0.60-0.70$ good $0.50-0.60$ moderate, $0.40-0.50$ fair and $<0.40$ weak or no association [20]. The level of significance was set at 0.05 . Statistical analyses were carried out using SAS Enterprise Guide 4.1 (SAS Institute, Inc., Cary, NC).

\section{Results}

\subsection{Face validity}

For the intra-session variability, the median IQR of the APS for every tasks ranged from $0.62^{\circ}$ to $1.35^{\circ}$, whereby $87.5 \%$ of all children had an $\mathrm{IQR}<2^{\circ}$. Median IQR ranged from $0.97^{\circ}$ to $1.20^{\circ}$ for the reach tasks $\left(88 \%<2^{\circ}\right)$, from $0.62^{\circ}$ to $1.08^{\circ}$ for the reach-tograsp tasks $\left(90 \%<2^{\circ}\right)$ and from $1.07^{\circ}$ to $1.35^{\circ}$ for the gross motor tasks $\left(83 \%<2^{\circ}\right)$.

Spearman-rank correlations between the Global-AVSs and Global-APS showed that none of the AVSs correlated high with the Global-APS (Table 1), except for the Global-AVS of wrist flexion ( $\rho$ 0.88). None of the Global-AVS pairs correlated highly with each other (all $\rho<0.60)$.

Correlations between the APS and AVSs per task were good to high for wrist flexion for all tasks ( $\rho 0.65-0.94)$, for elbow flexion in the reach-to-grasp tasks ( $\rho 0.61-0.78$ ), and elbow pro-supination during vertical grasping and hand to head ( $\rho 0.66-0.72)$. None of the AVS pairs correlated strongly with each other for the different tasks, except for elbow flexion and pro-supination during vertical grasping ( $\rho$ 0.69). 


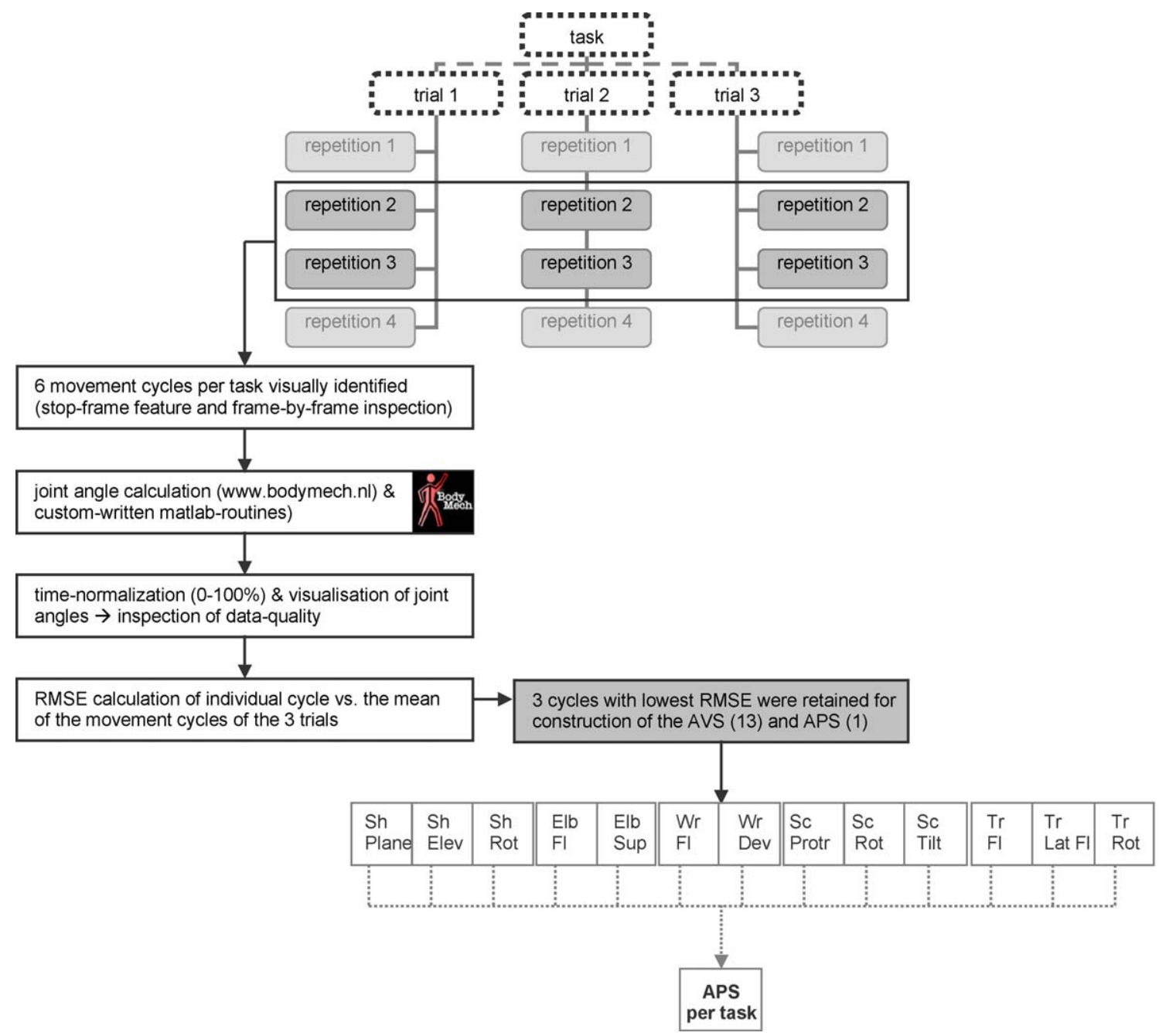

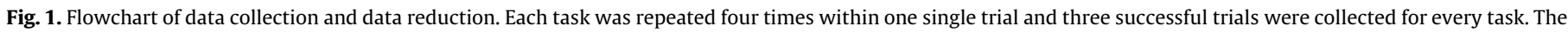

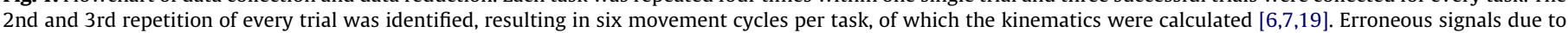

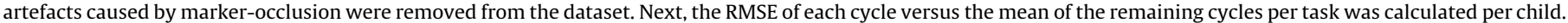

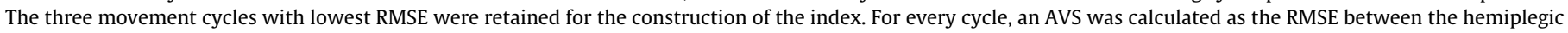

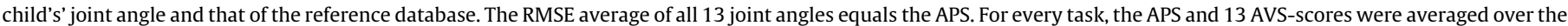

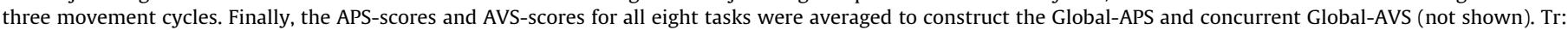
trunk; Sc: scapula; Sh: shoulder; Elb: elbow; Wr: wrist; RMSE: root mean square error; AVS: Arm Variable Score; APS: Arm Profile Score.

Table 1

Spearman rank correlations between the Global-APS and Global-AVS scores and between different pairs of Global-AVS scores.

\begin{tabular}{|c|c|c|c|c|c|c|c|c|c|c|c|c|c|}
\hline Global & Tr Fl & Tr Lat Fl & Tr Rot & Sc Protr & Sc Rot & Sc Tilt & Sh Plane & Sh Elev & Sh Rot & Elb Fl & Elb Pron & Wr Fl & Wr Dev \\
\hline APS & -0.09 & $0.49^{\circ}$ & $0.61^{*}$ & $0.46^{\circ}$ & -0.10 & 0.41 & 0.25 & 0.36 & 0.19 & 0.42 & 0.26 & $0.88^{\S}$ & -0.11 \\
\hline $\mathrm{Tr} \mathrm{Fl}$ & & -0.11 & -0.04 & 0.06 & -0.12 & 0.22 & 0.36 & 0.31 & -0.17 & 0.06 & 0.09 & -0.14 & 0.08 \\
\hline Tr Lat Fl & & & $0.48^{\circ}$ & -0.06 & 0.35 & $0.58^{*}$ & 0.04 & 0.06 & 0.04 & -0.03 & -0.09 & $0.52^{\circ}$ & 0.03 \\
\hline Tr Rot & & & & 0.13 & -0.06 & 0.39 & 0.05 & $0.46^{\circ}$ & 0.33 & 0.34 & 0.27 & 0.43 & 0.14 \\
\hline Sc Protr & & & & & 0.01 & -0.12 & 0.43 & -0.26 & 0.23 & $0.47^{\circ}$ & 0.43 & 0.21 & 0.08 \\
\hline Sc Rot & & & & & & 0.15 & -0.08 & -0.06 & -0.19 & -0.09 & -0.05 & 0.04 & 0.26 \\
\hline Sc Tilt & & & & & & & 0.23 & 0.35 & 0.15 & 0.15 & -0.21 & 0.37 & 0.10 \\
\hline Sh Plane & & & & & & & & -0.01 & 0.35 & 0.19 & -0.01 & 0.20 & 0.03 \\
\hline Sh Elev & & & & & & & & & -0.20 & 0.09 & 0.11 & 0.41 & 0.15 \\
\hline Sh Rot & & & & & & & & & & 0.25 & 0.24 & -0.06 & 0.17 \\
\hline Elb Fl & & & & & & & & & & & $0.45^{\circ}$ & 0.23 & -0.05 \\
\hline Elb Pron & & & & & & & & & & & & 0.08 & 0.22 \\
\hline Wr Fl & & & & & & & & & & & & & -0.25 \\
\hline
\end{tabular}

\footnotetext{
$\S<0.0001$

$* 0.01$.

o $<0.05$
} 

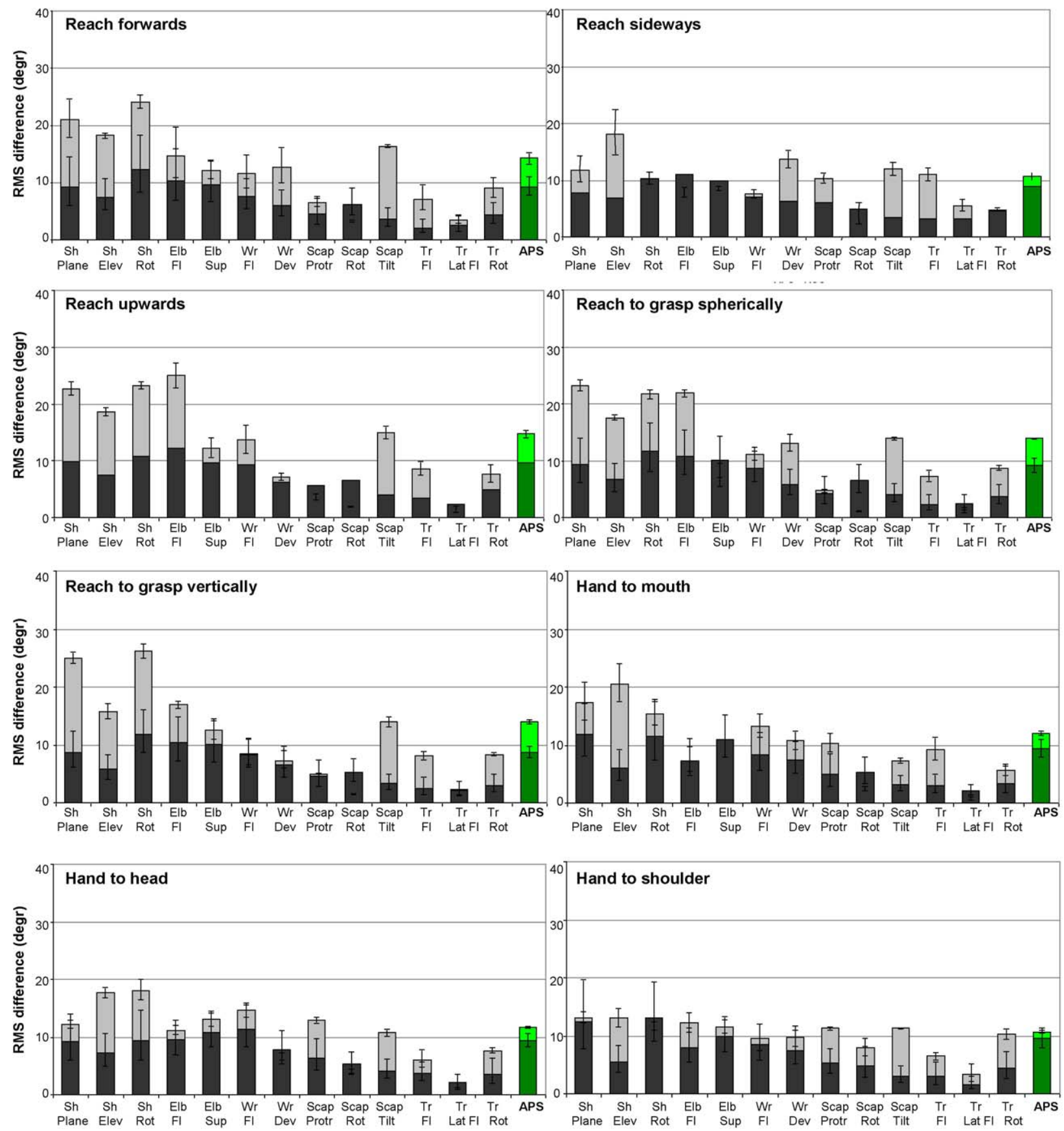

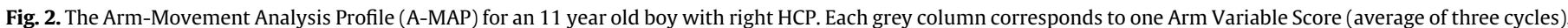

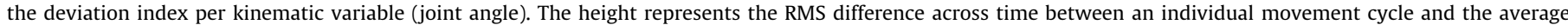

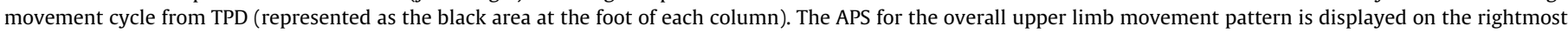
column, and is the average RMS difference across time for all kinematic variables. Tr: trunk; Sc: scapula; Sh: shoulder; Elb: elbow; Wr: wrist.

\subsection{Construct validity}

\subsubsection{House classification}

The distribution of the individual APS-scores per task, for the different categories of the House classification is given in Fig. 3. House scores for the children in the current study ranged from 4 to 7. Visual inspection shows decreasing APS-scores with increasing House-scores.

Table 2(A) shows significant good to high negative correlations between the House classification and the APS-scores, indicating that children with lower House-scores obtained higher APS-scores. For the Global-APS, a correlation of 0.80 was found. For the different tasks, coefficients ranged from 0.63 to 0.80 . Correlation analysis with the AVS-scores showed significant moderate to high negative correlations between the House-scores and the AVS of wrist flexion for all tasks ( $\rho$ 0.58-0.83). Moderate to good correlations were found with elbow flexion during the reach-tograsp tasks ( $\rho$ 0.59-0.69), and fair to moderate correlations with elbow pronation during vertical grasping and the gross motor tasks ( $\rho$ 0.46-0.55). Moderate correlations were also found with the 


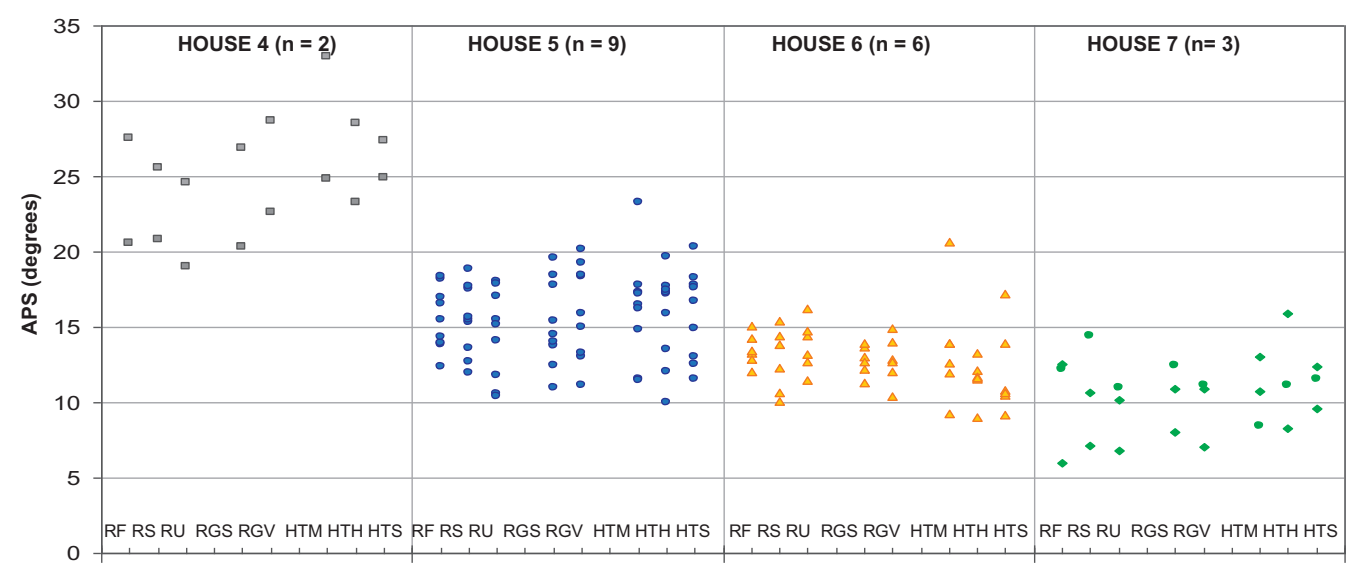

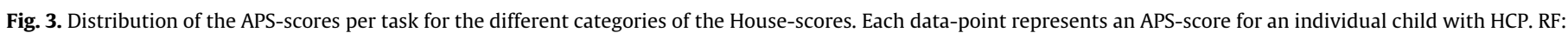

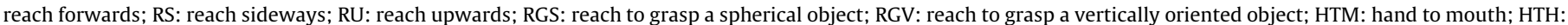
hand to head; HTS: hand to shoulder.

Table 2

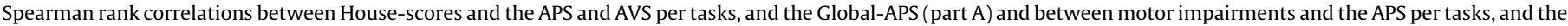
Global-APS (part B).

\begin{tabular}{|c|c|c|c|c|c|c|c|c|c|}
\hline & $\mathrm{RF}$ & RS & RU & RGS & RGV & HTM & HTH & HTS & Global \\
\hline \multicolumn{10}{|c|}{ (A) House classification } \\
\hline APS & $-0.78^{\S}$ & $-0.70^{¥}$ & $-0.63^{*}$ & $-0.77^{\S}$ & $-0.80^{\S}$ & $-0.68^{*}$ & $-0.71^{¥}$ & $-0.74^{¥}$ & $-0.80^{\S}$ \\
\hline Tr Fl & 0.05 & 0.09 & -0.09 & -0.13 & -0.17 & 0.11 & -0.37 & 0.15 & -0.00 \\
\hline Tr Lat Fl & 0.04 & -0.63 & -0.09 & -0.13 & -0.12 & -0.29 & -0.33 & -0.21 & -0.24 \\
\hline Tr Rot & -0.03 & -0.42 & -0.28 & -0.10 & -0.00 & -0.16 & $-0.56^{\circ}$ & -0.33 & -0.27 \\
\hline Sc Protr & -0.09 & -0.37 & -0.19 & -0.22 & -0.21 & -0.12 & -0.40 & -0.32 & -0.31 \\
\hline Sc Rot & 0.11 & 0.48 & -0.04 & 0.22 & -0.02 & -0.17 & -0.35 & 0.19 & 0.05 \\
\hline Sc Tilt & -0.31 & -0.12 & -0.30 & -0.42 & -0.35 & -0.18 & -0.22 & -0.06 & -0.35 \\
\hline Sh Plane & -0.15 & -0.14 & -0.16 & -0.10 & -0.22 & -0.18 & $-0.57^{*}$ & -0.40 & -0.23 \\
\hline Sh Elev & 0.07 & -0.13 & -0.26 & -0.33 & $-0.56^{\circ}$ & -0.25 & -0.25 & 0.03 & -0.34 \\
\hline Sh Rot & 0.10 & -0.09 & -0.02 & 0.08 & 0.06 & 0.09 & 0.07 & -0.28 & -0.01 \\
\hline Elb Fl & $-0.46^{\circ}$ & -0.29 & -0.40 & $-0.59^{*}$ & $-0.69^{¥}$ & -0.32 & -0.22 & -0.15 & $-0.49^{\circ}$ \\
\hline Elb Pron & 0.16 & -0.14 & -0.11 & -0.16 & $-0.55^{\circ}$ & $-0.49^{\circ}$ & $-0.46^{\circ}$ & $-0.50^{\circ}$ & -0.35 \\
\hline Wr Fl & $-0.73^{¥}$ & $-0.72^{¥}$ & $-0.68^{*}$ & $-0.72^{¥}$ & $-0.59^{*}$ & $-0.73^{¥}$ & $-0.58^{*}$ & $-0.83^{\S}$ & $-0.80^{\S}$ \\
\hline Wr Dev & 0.26 & 0.14 & 0.36 & 0.26 & 0.15 & 0.10 & 0.25 & 0.32 & 0.29 \\
\hline \multicolumn{10}{|c|}{ (B) Motor impairments } \\
\hline \multicolumn{10}{|c|}{ PROM } \\
\hline Total & 0.22 & 0.24 & 0.15 & 0.25 & 0.17 & -0.16 & 0.01 & 0.12 & 0.06 \\
\hline Shoulder & 0.05 & 0.01 & -0.00 & 0.12 & 0.02 & -0.19 & 0.01 & 0.15 & -0.03 \\
\hline Elbow & 0.15 & 0.26 & -0.02 & 0.11 & 0.18 & -0.16 & 0.16 & 0.04 & -0.01 \\
\hline Wrist & -0.01 & -0.03 & -0.03 & 0.08 & -0.09 & -0.15 & -0.34 & -0.23 & -0.14 \\
\hline \multicolumn{10}{|l|}{ MAS } \\
\hline Total & $0.52^{\circ}$ & 0.33 & 0.40 & $0.54^{\circ}$ & $0.45^{\circ}$ & 0.14 & 0.22 & 0.26 & 0.39 \\
\hline Shoulder & 0.28 & 0.13 & 0.05 & 0.30 & 0.27 & -0.01 & 0.16 & 0.14 & 0.15 \\
\hline Elbow & 0.05 & -0.15 & 0.09 & 0.03 & -0.03 & -0.24 & -0.12 & -0.16 & -0.07 \\
\hline Wrist & $0.69^{¥}$ & $0.66^{*}$ & $0.69^{¥}$ & $0.81^{\S}$ & $0.64^{*}$ & $0.56^{\circ}$ & 0.37 & $0.62^{*}$ & $0.73^{¥}$ \\
\hline \multicolumn{10}{|l|}{ MMT } \\
\hline Total & $-0.71^{¥}$ & $-0.48^{\circ}$ & $-0.57^{*}$ & $-0.70^{¥}$ & $-0.65^{*}$ & -0.43 & $-0.48^{\circ}$ & $-0.57^{*}$ & $-0.65^{*}$ \\
\hline Shoulder & -0.31 & -0.27 & -0.22 & -0.27 & -0.44 & -0.21 & -0.20 & -0.24 & -0.35 \\
\hline Elbow & $-0.57^{*}$ & -0.32 & $-0.49^{\circ}$ & $-0.61^{*}$ & -0.44 & -0.22 & -0.27 & -0.40 & $-0.48^{\circ}$ \\
\hline Forearm & $-0.72^{¥}$ & $-0.54^{\circ}$ & $-0.63^{*}$ & $-0.76^{\S}$ & $-0.71^{¥}$ & $-0.58^{*}$ & $-0.58^{*}$ & $-0.62^{*}$ & $-0.71^{¥}$ \\
\hline Wrist & $-0.64^{*}$ & -0.40 & -0.39 & $-0.70^{¥}$ & $-0.57^{*}$ & -0.30 & -0.43 & $-0.45^{\circ}$ & $-0.53^{\circ}$ \\
\hline Grip ratio & $-0.67^{*}$ & $-0.55^{\circ}$ & $-0.56^{\circ}$ & $-0.73^{¥}$ & $-0.70^{¥}$ & $-0.60^{*}$ & $-0.48^{\circ}$ & $-0.66^{*}$ & $-0.66^{*}$ \\
\hline
\end{tabular}

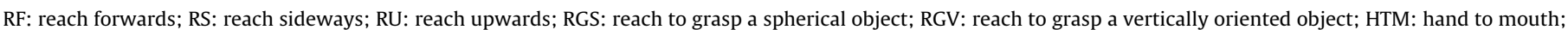
HTH: hand to head; HTS: hand to shoulder. PROM: passive range of motion; MAS: modified Ashworth Scale for Spasticity; MMT: manual muscle testing.

$\S<0.0001$

$¥<0.001$.

$<0.01$.

$\circ<0.05$

shoulder elevation plane and trunk rotation during hand to head ( $\rho$ $0.56-0.57)$ and with shoulder elevation during vertical grasping ( $\rho$ $0.56)$.

\subsubsection{Motor impairments}

Table 2(B) shows the Spearman-rank correlations between the measures of motor impairment and the APS. No significant correlations were found for PROM. Moderate to high correla- tions were found between muscle tone at the wrist and the Global-APS and APS for all but one task ( $\rho$ 0.56-0.81). Moderate to high correlations were found between muscle strength of the forearm, grip strength and the Global-APS and APS for the reach and reach-to-grasp tasks ( $\rho$ 0.54-0.76). Total strength and wrist strength correlated good to high with the APS for forward reaching and spherical grasping ( $\rho$ 0.64-0.71). 


\section{Discussion}

This paper introduces the "Arm Profile Score" (APS) to grade the overall severity of UL movement pathology, based on objective data from 3DMA. The APS can be decomposed into 13 Arm Variable Scores (AVS), representing the different joint angles. The APS, together with the AVSs form the "Arm Movement Analysis Profile" (A-MAP). As for the GPS, these indices are reported in the same units as the kinematic variables (degrees), supporting an easy interpretation [10]. Still, the magnitude of the reported AVS should always be related to the expected individual joint ROM and to the kinematic graphs, as the AVS-scores do not provide an indication of the direction of the pathological joint movement [10].

As gait is a well-defined, cyclic movement representing the main lower limb function, gait pathology can be expressed by a single index, the GPS. In contrast, UL movements are complex, noncyclic and non-repetitive by nature. Moreover, as there is no single most important UL function, several clinically relevant tasks need to be assessed, resulting in multiple APS-scores. Nonetheless, to provide the clinician with one deviation index, the Global-APS was also calculated, being the average APS of all tasks. It is however important to notice that there was no pre-selection of any specific kinematic variable or a weighing of variables that are thought to contribute more to a deviating movement pattern. Further refinement of the Global-APS would require the identification of task-dependent kinematic parameters. Such fine-tuning, based on a specific task-selection per joint, could improve the value of the index as a summary measure of UL movement pathology.

The construction of the APS as a Euclidean distance measure inherently confirms the face validity [10]. This was further established through the analysis of intra-session variability. The median IQR for all tasks was below $2^{\circ}$. These low IQR indicate that the APS can be used as a reliable measure within a single session for the different tasks, when based on the three most representative movement cycles. Previous research has also shown that the average measurement error within one session for UL angular waveforms ranged between $3.2^{\circ}$ and $5.5^{\circ}$ for the different tasks [7], hereby confirming the reliability of the measurement procedure and outcome variables.

Although the APS and AVS are mathematically related, there were no high correlations between the individual AVS and the APS, or between pairs of AVS-scores. This suggests that there is considerably more information enclosed within the A-MAP than in the APS alone and that the A-MAP may provide useful insights into which joint angles are contributing to an elevated APS for each of the tasks. Only for the AVS of wrist flexion, significant high correlations were found with the APS, stressing the importance of the wrist position in overall task performance.

To test the construct validity, the APS was related to other UL measures. To ensure that all children were able to perform the complete movement protocol, this study included only children with a minimum ability to actively grasp and hold an object weakly (House 4). Nonetheless, current results already showed highly significant correlations between the House-scores and the APS, providing evidence of its construct validity. Also, House-scores were highly correlated with the AVS-scores of wrist flexion and elbow pro-supination, which might point to the importance of adequate elbow supination and wrist mobility for the functional abilities of the UL.

The House-scores [12], rather than the MACS were used as a functional classification. Although the MACS [13] is recently considered the appropriate measure to classify a child's UL abilities, the distribution of the scores within our study sample did not allow further comparisons between the APS and the MACS. Still, further study is needed including a larger group of children sampled across the different MACS-levels.
Correlation analysis additionally showed that increased wrist tone, decreased forearm strength and lower grip strength were highly correlated with higher APS-scores, especially for the reach and reach-to-grasp tasks. Current results confirm the role of distal muscle tone and strength in the emergence of UL kinematic deficits. This suggests that treatment aimed at distal tone reduction, e.g. BTX-injections, or strength training, might influence the AVS-scores. The high correlations found between the APS and underlying motor impairments contrast the lower correlations that have been reported between lower limb muscle tone and strength and single joint kinematic gait parameters [21,22]. Thus far, correlations between the GPS and underlying impairments have not yet been reported, though significant correlations between the GPS and clinical judgement of severity of gait pathology have been well established [23].

This study proposed the APS, a summary index to grade the severity of UL movement pathology based on kinematic data. This index may aid in the interpretation of the complex and highly interdependent graphs, as it can be decomposed into AVSs, representing deviating indices for the different UL joint angles. Results showed good correlations between the APS and other UL measures, providing a sound base for its use as a tool to evaluate the severity of movement pathology in hemiplegic children. Further study will need to confirm its value as an outcome measurement, as well as its applicability for other pathologies, e.g. quadriplegic CP or Erb's-palsy.

\section{Acknowledgement}

Ellen Jaspers received a PhD fellowship of the Research Foundation-Flanders (FWO), Belgium.

\section{Conflict of interest statement}

The authors report no financial or personal relationships with other people or organizations that could inappropriately influence their work.

\section{References}

[1] Klingels K, Jaspers E, Van de Winckel A, De Cock P, Molenaers G, Feys H. A systematic review of arm activity measures for children with hemiplegic cerebral palsy. Clin Rehabil 2010;24:887-900.

[2] Butler EE, Lad AL, Louie SA, LaMont LE, Wong W, Rose J. Three-dimensional kinematics of the upper limb during a reach and grasp cycle for children. Gait Posture 2010;32:72-7.

[3] Mackey AH, Miller F, Walt SE, Waugh MC, Stott NS. Use of three-dimensional kinematic analysis following upper limb botulinum toxin A for children with hemiplegia. Eur J Neurol 2008;15:1191-8.

[4] Jaspers E, Desloovere K, Bruyninckx H, Molenaers G, Klingels K, Feys H. Review of quantitative measurements of upper limb movements in hemiplegic cerebral palsy. Gait Posture 2009;30:395-404.

[5] Wu G, van der Helm FCT, Veeger HEJ, Makhsous M, Van Roy P, Anglin C, et al. ISB recommendation on definitions of joint coordinate systems of various joints for the reporting of human joint motion. Part II. Shoulder, elbow, wrist and hand. J Biomech 2005;38:981-92.

[6] Jaspers E, Feys H, Bruyninckx H, Harlaar J, Molenaers G, Desloovere K. Upper limb kinematics: development and reliability of a clinical protocol for children. Gait Posture 2011;33:279-85.

[7] Jaspers E, Feys H, Bruyninckx H, Cutti AG, Harlaar J, Molenaers G, Desloovere K. The reliability of upper limb kinematics in children with hemiplegic cerebral palsy. Gait Posture 2011;33:568-75.

[8] Schutte LM, Narayanan U, Stout JL, Selber P, Gage JR, Schwartz MH. An index for quantifying deviations from normal gait. Gait Posture 2000;11:25-31.

[9] Schwartz MH, Rozumalski A. The gait deviation index: a new comprehensive index of gait pathology. Gait Posture 2008;28:351-7.

[10] Baker R, McGinley JL, Schwartz MH, Beynon S, Rozumalski A, Graham HK, et al. The gait profile score and movement analysis profile. Gait Posture 2009;30:265-9.

[11] Riad J, Coleman S, Lundh D, Broström E. Arm posture score and arm movement during walking: a comprehensive assessment in spastic hemiplegic cerebral palsy. Gait Posture 2011;33:48-53.

[12] House JH, Gwathmey FW, Fidler MO. A dynamic approach to the thumb-in palm deformity in cerebral palsy. J bone Joint Surg Am 1981;63:216-25. 
[13] MACS. Manual Ability Classification System. MACS 2005. Available from: http://www.macs.nu/.

[14] Klingels K, De Cock P, Molenaers G, Desloovere K, Huenaerts C, Jaspers E, et al Upper limb motor and sensory impairments in children with hemiplegic cere bral palsy. Can they be measured reliably? Disabil Rehabil 2010;32:409-16.

[15] Woltring HJ. Smoothing and differentiation techniques applied to 3-D data. In: Allard P, Stokes IAF, Blanchi J-P, editors. Three-dimensional analysis of human movement. Champaign: Human Kinetics Publishers; 1995.

[16] Bohannon RW, Smith MB. Interrater reliability of a modified Ashworth scale of muscle spasticity. Phys Ther 1987;67:206-7.

[17] Hislop HJ, Montgomery J. Daniels and Worthingham's muscle testing: techniques of manual examination.. Philadelphia: Harcourt Brace \& Company; 1995

[18] Petuskey K, Bagley A, Abdala E, James MA, Rab G. Upper extremity kinematics during functional activities: three-dimensional studies in a normal pediatric population. Gait Posture 2007;25:573-9.
[19] van Andel CJ, Wolterbeek N, Doorenbosch CA, Veeger DH, Harlaar J. Complete 3D kinematics of upper extremity functional tasks. Gait Posture 2008;27: 120-7.

[20] Hinkle DE, Wiersma W, Jurs SG. Applied statistics for the behavioural sciences, 4th ed., Boston: Houghton Mifflin Company; 1998.

[21] Desloovere K, Molenaers G, Feys H, Huenaerts C, Callewaert B, Van de Walle P. Do dynamic and static clinical measurements correlate with gait analysis parameters in children with cerebral palsy? Gait Posture 2006;24:302-13.

[22] Abel MF, Damiano DL, Blanco JS, Conaway M, Miller F, Dabney K, et al. Relationships among musculoskeletal impairments and functional health status in ambulatory cerebral palsy. J Pediatr Orthop 2003;23:341-535.

[23] Beynon S, McGinley JL, Dobson F, Baker R. Correlations of the gait profile score and the movement analysis profile relative to clinical judgments. Gait Posture 2010;32:129-32. 